\title{
Novos compostos de milho-pipoca para o Brasil
}

\section{New composites of popcorn to Brazil}

\author{
Carlos Alberto Scapim ${ }^{1 *}$; Antônio Teixeira do Amaral Júnior ${ }^{2}$; \\ Rafael Augusto Vieira ${ }^{3}$; Lia Mara Moterle ${ }^{4}$; Leandra Regina Texeira ${ }^{4}$; \\ Joselaine Viganó ${ }^{5}$; Gilberto Barbosa Sandoval Júnior ${ }^{6}$
}

\section{Resumo}

Apesar dos avanços, o número de variedades e híbridos comerciais de linhagens de milho-pipoca é reduzido. Assim, o objetivo deste trabalho foi avaliar novos compostos desenvolvidos no Brasil e indicar os de maior potencial genético para iniciar um trabalho de melhoramento intrapopulacional. A pesquisa foi desenvolvida no ano agrícola 2005/2006. Os tratamentos constaram de 21 genótipos (híbridos de linhagens, compostos e sintéticos) cultivados em dois municípios, Umuarama e Cidade Gaúcha, ambos no Noroeste do Estado do Paraná. As características avaliadas por meio de análises de variância individuais e conjunta foram rendimento de grãos $(R G)$ e capacidade de expansão (CE). Os compostos UEM 5, UEM 6 e UEM 7 expressaram alta qualidade da pipoca (CE), com valores semelhantes às testemunhas em ambos os locais. O rendimento dos genótipos foi mais instável do que a capacidade de expansão entre os locais avaliados. Os compostos UEM 5, UEM 6 e UEM 7 revelaram potencial satisfatório tanto para CE quanto para RG quando avaliados em Umuarama, PR.

Palavras-chave: Zea mays, rendimento de grãos, capacidade de expansão

\begin{abstract}
Although advances are obtained, the number of varieties and hybrids from inbred lines of popcorn are reduced. The objective of this work was to evaluate new composites developed in Brazil and to indicate those which present better potential to initiate an intrapopulation breeding program. Trials were carried out during 2005/2006 cropping season. Treatments included 21 genotypes (hybrid from inbred lines, composites and synthetic) cultivated in two locations (Umuarama and Cidade Gaúcha), both located in Northwest of Paraná State. Grain yield (GY) and popping expansion (PE) were evaluated by individual and joint analyses of variance. Composites UEM 5, UEM 6 and UEM 7 obtained the highest quality of the PE, with similar values to the check treatments in both locations. GY of genotypes ranged more than popping expansion over locations. Composites UEM 5, UEM 6 and UEM 7 revealed satisfactory potential both PE and GY when evaluated in Umuarama, Paraná State, Brazil.
\end{abstract}

Key words: Zea mays, composites, grain yield, popping expansion

\footnotetext{
1 Eng. Agr., Prof. Dr, Departamento de Agronomia, Universidade Estadual de Maringá (UEM), Maringá, PR. E-mail: cascapim@, uem.br.

2 Eng. Agr., Prof. Dr, Universidade Estadual do Norte Fluminense Darcy Ribeiro, Campos dos Goytacazes, SP. E-mail: amaraljr@ uenf.br

3 Eng. Agr., Mestrando pelo Programa de Pós-graduação em Genética e Melhoramento, Universidade Estadual de Maringá (UEM). E-mail: rfavieira@msn.com

4 Eng. Agr., Doutor em Agronomia. E-mail: lmoterle@hotmail.com, leandra_texeira@yahoo.com.br

5 Bióloga, M. Sc. em Genética e Melhoramento, Doutoranda pelo Programa de Pós-graduação em Genética e Melhoramento, Universidade Estadual de Maringá (UEM). E-mail: josyvigano@yahoo.com.br

6 Eng. Agr. E-mail: gbs_jr@hotmail.com

* Autor para correspondência
} 


\section{Introdução}

Na safra agrícola de 2008/09, 302 cultivares de milho estiveram disponibilizadas para comercialização, sendo doze de milhos especiais (sete cultivares de milho-pipoca, duas de milho doce, uma de milho ceroso e duas para utilização de canjica). Quarenta e seis cultivares foram lançadas, substituindo 22 que deixaram de ser comercializadas; demonstrando assim a dinâmica dos programas de melhoramento, a confiança do setor na evolução da cultura e a importância do uso de semente no aumento da produtividade (CRUZ; PEREIRAFILHO, 2008). As sete cultivares de milho-pipoca são: Zélia (híbrido triplo), Jade (híbrido triplo), IAC 112 (híbrido simples modificado), IAC 125 (híbrido triplo), BRS Angela (variedade), RS-20 (variedade) e UFVM2-Barão-Viçosa (variedade). Verificase, portanto, que apesar dos avanços o número de variedades e híbridos de linhagens comerciais de milho-pipoca, é reduzido em relação à crescente demanda pelo produto (VENDRUSCOLO et al., 2001; MATTA; VIANA, 2001; ANDRADE et al., 2002; DAROS et al., 2004; FREITAS JÚNIOR et al., 2006; SCAPIM et al., 2006; RANGEL et al., 2007; RANGEL et al., 2008; SANTOS et al., 2008; VIEIRA et al., 2009).

$\mathrm{Na}$ Região Noroeste do Paraná existem empacotadoras que comprariam o produto se os agricultores começassem a semear em larga escala (PINTO et al., 2007). No entanto, é muito difícil conseguir sementes disponíveis no mercado para o ano agrícola.

Contrastando com o milho comum, o número de cultivares do milho-pipoca disponível no mercado brasileiro é pequeno, fazendo com que o melhoramento intrapopulacional seja opção de desenvolvimento de novas variedades para uso per se, além de permitir a estimação de parâmetros genéticos e componentes de variâncias, praticamente inexistentes na literatura sobre essa cultura; também, pode facilitar os futuros trabalhos de extração de linhagens endogâmicas decorrentes do incremento que a seleção recorrente provoca nas freqüências dos alelos favoráveis (HALLAUER; MIRANDA FILHO, 1988; PEREIRA; AMARAL JÚNIOR, 2001; VILARINHO et al., 2003; VIANA et al., 2007).

Antesdeiniciaromelhoramentointrapopulacional é fundamental saber quais os genótipos que serão trabalhados geneticamente. A formação de compostos de alta variabilidade e potencial pode indicar o sucesso do melhoramento com os métodos de melhoramento intrapopulacionais consagrados.

Trabalhando com a população UNB-2U, de milho-pipoca, Pereira e Amaral Júnior (2001), por meio do Delineamento I, constataram que, em ordem, os maiores ganhos seriam possíveis com a aplicação das metodologias intrapopulacionais de seleção entre famílias $\mathrm{S}_{1}$, bem como entre famílias de irmãos completos, entre famílias de meiosirmãos e de seleção entre famílias por seleção massal estratificada.

A capacidade de expansão, produtividade e tolerância às doenças têm sido consideradas as característicasdemaiorimportâncianomelhoramento do milho-pipoca. Avaliar corretamente os genótipos para essas características é de fundamental importância. A ampla variabilidade genética verificada em muitas populações (MIRANDA et al., 2007) conduz à hipótese de que há possibilidade de seleção de material com alta capacidade de expansão, produtividade elevada e resistência às doenças (CARPENTIERI-PÍPOLO et al., 2003).

Por conseguinte, o objetivo deste trabalho foi avaliar e identificar novos compostos desenvolvidos no Brasil, bem como indicar os de maior potencialidade genética para iniciar trabalhos de melhoramento intrapopulacional.

\section{Material e Métodos}

A avaliação dos genótipos (híbridos de linhagens, compostos e sintéticos) foi realizada em experimentos instalados nos municípios de 
Umuarama(Fazenda Experimental de Umuarama, na UEM, PR) e Cidade Gaúcha (Fazenda Experimental da Universidade Estadual de Maringá, Campus do Arenito), ambos os municípios situados na região Noroeste do Estado do Paraná. Essa região apresenta clima mesotérmico úmido, com chuvas de verão e de outono, e verão quente (GODOY; CORREA; SANTOS, 1976). O Latossolo Vermelho Distrófico representa a unidade de solo predominante nas áreas experimentais (EMBRAPA, 1999).

O município de Umuarama situa-se a 403 metros de altitude, entre a latitude $23^{\circ} 47^{\prime}$ Sul e Longitude $53^{\circ} 14^{\prime}$ Oeste. O município de Cidade Gaúcha situa- se a 550 metros de altitude, entre a latitude $23^{\circ} 22^{\prime}$ Sul e longitude 52 56' Oeste (IAPAR, 1987).

Ambos os experimentos foram instalados em blocos completos com os tratamentos ao acaso com três repetições, durante o ano agrícola 2005/2006. Os genótipos avaliados estão descritos na (Tabela 1). É muito importante ressaltar que os compostos da UEM são medianamente tolerantes à mancha branca (Phaeosphaeria maydis), helmintosporiose (Exserohilum turcicum), cercosporiose (Cercospora zeae-maydis) e ferrugem polissora (Puccinia polysora).

Tabela 1. Caracterização dos genótipos avaliados em dois locais do Noroeste do Paraná no ano agrícola 2005/2006.

\begin{tabular}{|c|c|c|c|c|}
\hline Genótipos & Tipo & Origem & Genealogia & $\begin{array}{c}\text { Cor dos } \\
\text { grãos } \\
\end{array}$ \\
\hline IAC 112 & $\begin{array}{l}\text { Híbrido simples } \\
\text { modificado }\end{array}$ & IAC & Guarani e IAC 64 & Amarelos \\
\hline Zélia original & Híbrido triplo & Pioneer & Desconhecida & Amarelos \\
\hline BRS Ângela & Variedade & EMBRAPA & CMS-43 & Brancos \\
\hline IAC 125 & Híbrido simples & IAC & Desconhecida & Amarelos \\
\hline IAC 1283 & Híbrido simples & IAC & Desconhecida & Amarelos \\
\hline $\mathrm{SC} 002$ & Variedade & UEL & Geração avançada de híbridos americanos & Brancos \\
\hline Viçosa & Variedade & UFV & Desconhecida & Amarelos \\
\hline Beija Flor & Variedade & UFV & Desconhecida & Amarelos \\
\hline PR 038 & Variedade & UEL & Geração avançada de híbridos americanos & Brancos \\
\hline PR 079 & Variedade & UEL & Geração avançada de híbridos americanos & Brancos \\
\hline RR 046 & Variedade & UEL & Geração avançada de híbridos americanos & Brancos \\
\hline SC 016 & Variedade & UEL & Geração avançada de híbridos americanos & Brancos \\
\hline PR 017 & Variedade & UEL & Geração avançada de híbridos americanos & Brancos \\
\hline UNB 2 & Variedade & UNB & $\begin{array}{l}\text { Originou-se do Composto Indígena da } \\
\text { ESALQ-USP }\end{array}$ & Amarelos \\
\hline UEM 1 & Composto & UEM & $\begin{array}{l}\text { Formado a partir de cruzamentos entre } \\
\text { populações locais e híbridos norte-americanos }\end{array}$ & Amarelos \\
\hline UEM 2 & Sintético & UEM & $\begin{array}{l}\text { Formado a partir de linhagens } \mathrm{S}_{5} \text { de alta } \\
\text { capacidade geral de combinação do híbrido } \\
\text { triplo Zélia }\end{array}$ & Amarelos \\
\hline UEM 3 & Composto & UEM & $\begin{array}{l}\text { Cruzamento entre IAC 112, Zélia e Ângela e } \\
\text { posterior recombinação }\end{array}$ & Amarelos \\
\hline UEM 4 & Composto & UEM & $\begin{array}{l}\text { Composto originado de oito populações de } \\
\text { grãos brancos }\end{array}$ & Brancos \\
\hline UEM 5 & Composto & UEM & $\begin{array}{l}\text { Originado de gerações avançadas de híbridos } \\
\text { americanos e o IAC } 112\end{array}$ & Amarelos \\
\hline UEM 6 & Composto & UEM & $\begin{array}{l}\text { Originado de gerações avançadas de híbridos } \\
\text { americanos e a variedade Angela }\end{array}$ & Brancos \\
\hline UEM 7 & Composto & UEM & $\begin{array}{l}\text { Originado de gerações avançadas de híbridos } \\
\text { americanos, o IAC } 112 \text { e Zélia }\end{array}$ & Amarelos \\
\hline
\end{tabular}


Cada parcela foi representada por duas fileiras de 5,00 m de comprimento, com espaçamento de 0,90 $\mathrm{m}$ entre fileiras. A semeadura foi realizada em covas espaçadas a $0,20 \mathrm{~m}$, semeando-se duas sementes por cova e deixando-se apenas uma plântula após o desbaste. $\mathrm{O}$ desbaste foi realizado entre 20 e 25 dias após a emergência das plântulas. A adubação de base foi realizada com a aplicação de $400,00 \mathrm{~kg} \mathrm{ha}^{-1}$ do adubo formulado 4-14-8 + Zn, sendo aplicada uma dosagem de $150,00 \mathrm{~kg} \mathrm{ha}^{-1}$ de uréia em adubação de cobertura 30 dias após a emergência. Os tratos culturais aplicados seguiram as práticas comumente adotadas na cultura, em ambos os experimentos.

Por ocasião da colheita, o rendimento de grãos foi avaliado mediante a pesagem da massa de grãos debulhados de cada parcela, seguida pela conversão do rendimento a $\mathrm{kg} \mathrm{ha}^{-1}$ e ajuste da massa para a umidade padrão de $13 \%$, com correção de estande para um padrão de 50 plantas por parcela mediante a técnica de análise de covariância indicada por Vencovsky e Barriga (1992).

A capacidade de expansão foi mensurada em laboratório pelo cálculo da razão entre o volume da pipoca expandida e a massa de grãos crus. Foram tomados os dados obtidos de duas amostras de $30 \mathrm{~g}$ de grãos por parcela. Cada amostra foi estourada em uma pipoqueira elétrica com controle automático de temperatura, desenvolvida pelo Centro Nacional de Instrumentação Agrícola da EMBRAPA. A umidade do estouro foi de $13 \%$. As amostras foram submetidas a uma temperatura constante de 280 ${ }^{\circ} \mathrm{C}$ por um período de dois minutos. O volume de pipoca expandida foi medido em proveta graduada de $2.000 \mathrm{~mL}$.

Os dados de rendimento de grãos e de capacidade de expansão foram submetidos às análises de variância individuais e conjunta (CRUZ; CARNEIRO, 2003). As médias foram comparadas pelo teste de agrupamento de médias proposto por Scott e Knott (1974), considerando um nível de $5 \%$ de probabilidade. As análises estatísticas foram realizadas utilizando-se o programa computacional Sisvar (FERREIRA, 2003).

\section{Resultados e Discussão}

Uma vez que o teste $\mathrm{F}$ máximo não revelou, a $5 \%$ de probabilidade, heterogeneidade entre os quadrados médios do resíduo das análises individuais para capacidade de expansão $(\mathrm{CE})$ e rendimento de grãos $(\mathrm{RG})$ foi possível a realização das análises de variância conjunta.

Pela análise de variância conjunta, observouse que houve diferenças significativas, em nível de $5 \%$ de probabilidade, para os efeitos principais genótipos e locais, bem como para a interação genótipos versus locais (Tabela 2). A média de RG (2.443,00 $\left.\mathrm{kg} \mathrm{ha}^{-1}\right)$ dos 21 genótipos em Umuarama foi superior do que Cidade Gaúcha $(1.813,00$ $\mathrm{kg} \mathrm{ha} \mathrm{h}^{-1}$ ). A explicação mais plausível foi o déficit hídrico na fase de florescimento que prejudicou o rendimento médio dos genótipos em Cidade Gaúcha. Os resultados indicam que, de maneira geral, os genótipos comportam-se diferencialmente frente aos dois locais, justificando o estudo do comportamento dos genótipos em cada local.

Para CE, o coeficiente de variação foi de $16,6 \%$, enquanto que para RG o valor obtido foi de $31,23 \%$ (Tabela 2). Os valores referentes ao CV encontrados nesse trabalho estão acima dos observados por Galvão, Sawazaki e Miranda (2000), os quais obtiveram para CE, $1,86 \%$ e RG, $12,86 \%$. Sawazaki et al. (2000) testando o potencial de linhagens, também observaram baixos valores de CE. Por outro lado, o valor de CV para CE obtido nesse trabalho foi inferior ao observado por Carpentieri-Pípolo, Silva e Seifert (2002), os quais encontraram CV de $21,11 \%$ trabalhando também com milho-pipoca. 
Tabela 2. Análise de variância conjunta para capacidade de expansão $\left(\mathrm{mL} \mathrm{g}^{-1}\right)$ e rendimento $\left(\mathrm{kg} \mathrm{ha}^{-1}\right)$, proveniente de experimentos com 21 genótipos de milho-pipoca em dois locais, na safra de 2005/2006.

\begin{tabular}{lccc}
\hline \multirow{2}{*}{ Fonte de variação } & \multirow{2}{*}{ GL } & \multicolumn{2}{c}{ QM } \\
\cline { 3 - 4 } & & Capacidade de expansão $\left(\mathbf{m L ~ ~ ^ { - 1 } )}\right.$ & Rendimento de grãos (kg ha $\left.^{-1}\right)$ \\
\hline Genótipos (G) & 20 & $209,00^{*}$ & $5.052 .976,98^{*}$ \\
Locais (L) & 1 & $44,98^{\mathrm{ns}}$ & $16.655 .313,14^{*}$ \\
Blocos/Locais (B/L) & 6 & 6,23 & $1.218 .917,00$ \\
G x L & 20 & $37,91^{*}$ & $1.159 .976,76^{*}$ \\
Resíduo & 120 & 18,33 & $441.694,11$ \\
\hline CV (\%) & & 16,66 & 31,23 \\
Média geral & 25,70 & 2.128 \\
\hline
\end{tabular}

${ }^{*}$ significativo a $5 \%$ de probabilidade; ${ }^{\text {ns }}$ não-significativo a $5 \%$ de probabilidade.

Em relação ao $\mathrm{CV}$ para rendimento de grãos $(31,23 \%)$, provavelmente, o valor encontrado se deve a alta variabilidade existente nos materiais avaliados, mas sobretudo por se tratar de característica quantitativa, por conseguinte, mais sensível aos efeitos ambientais (SCAPIM; CARVALHO; CRUZ, 1995).
Os resultados médios dos 21 genótipos avaliados, quanto à CE e RG em Umuarama e Cidade Gaúcha (Tabela 3) revelaram que os efeitos entre os genótipos em um mesmo local e em locais diferentes foram significativos $(\mathrm{P}<0,05)$ pelo teste de médias e teste $\mathrm{F}$ para as características avaliadas. Esses resultados indicam a existência de variabilidade entre os genótipos estudados, de fundamental importância para a aplicação da seleção.

Tabela 3. Valores médios da capacidade de expansão $\left(\mathrm{mL} \mathrm{g}^{-1}\right)$ e rendimento de grãos $\left(\mathrm{kg} \mathrm{ha}^{-1}\right)$, proveniente de experimentos com 21 genótipos de milho-pipoca em dois locais, na safra de 2005/2006.

\begin{tabular}{|c|c|c|c|c|}
\hline \multirow{2}{*}{ Genótipos } & \multicolumn{2}{|c|}{ Capacidade de expansão $\left(\mathrm{mL} \mathrm{g}^{-1}\right)$} & \multicolumn{2}{|c|}{ Rendimento degrãos $\left(\mathrm{kg} \mathrm{ha}^{-1}\right)$} \\
\hline & Umuarama & Cidade Gaúcha & Umuarama & Cidade Gaúcha \\
\hline IAC 112 & $28,95 \mathrm{aA}$ & $34,08 \mathrm{aA}$ & $2.632,00 \mathrm{cA}$ & $2.338,00 \mathrm{bA}$ \\
\hline Zélia original & $34,29 \mathrm{aA}$ & $30,37 \mathrm{aA}$ & $1.866,00 \mathrm{cA}$ & $2.376,00 \mathrm{bA}$ \\
\hline BRS Angela & $26,41 \mathrm{aA}$ & $31,00 \mathrm{aA}$ & $1.356,00 \mathrm{dA}$ & $2.047,00 \mathrm{bA}$ \\
\hline IAC 125 & $33,87 \mathrm{aA}$ & $33,29 \mathrm{aA}$ & $4.868,00 \mathrm{aA}$ & $2.962,00 \mathrm{aB}$ \\
\hline IAC 1283 & $32,29 \mathrm{aA}$ & $34,46 \mathrm{aA}$ & $2.850,00 \mathrm{bA}$ & $3.539,00 \mathrm{aA}$ \\
\hline SC 002 & $25,12 \mathrm{bA}$ & $19,87 \mathrm{bA}$ & $1.700,00 \mathrm{cA}$ & $2.117,00 \mathrm{bA}$ \\
\hline Viçosa & $25,54 \mathrm{bA}$ & $27,37 \mathrm{aA}$ & $2.350,00 \mathrm{cA}$ & $922,00 \mathrm{~dB}$ \\
\hline Beija-flor & $24,37 \mathrm{bA}$ & $19,96 \mathrm{bB}$ & $2.403,00 \mathrm{cA}$ & $1.345,00 \mathrm{cB}$ \\
\hline PR 038 & $16,21 \mathrm{cB}$ & $23,45 \mathrm{bA}$ & $1.916,00 \mathrm{cA}$ & $892,00 \mathrm{~dB}$ \\
\hline PR 079 & $16,83 \mathrm{cB}$ & $23,54 \mathrm{bA}$ & $2.341,00 \mathrm{cA}$ & $1.680,00 \mathrm{cA}$ \\
\hline RR 046 & $12,25 \mathrm{cB}$ & $22,04 \mathrm{bA}$ & $883,00 \mathrm{dA}$ & $967,00 \mathrm{dA}$ \\
\hline SC 016 & $22,16 \mathrm{bA}$ & $22,91 \mathrm{bA}$ & $1.191,00 \mathrm{dA}$ & $631,00 \mathrm{dA}$ \\
\hline PR 017 & $21,20 \mathrm{bA}$ & $21,66 \mathrm{bA}$ & $2.170,00 \mathrm{cA}$ & $488,00 \mathrm{~dB}$ \\
\hline UNB 2 & $24,54 \mathrm{bA}$ & $19,91 \mathrm{bA}$ & $4.012,00 \mathrm{aA}$ & $2.580,00 \mathrm{bB}$ \\
\hline UEM 1 & $19,37 \mathrm{cA}$ & $23,54 \mathrm{bA}$ & $2.339,00 \mathrm{cA}$ & $1.728,00 \mathrm{cA}$ \\
\hline UEM 2 & $30,87 \mathrm{aA}$ & $25,12 \mathrm{bA}$ & $1.942,00 \mathrm{cA}$ & $1.577,00 \mathrm{cA}$ \\
\hline UEM 3 & $22,79 \mathrm{bA}$ & $22,95 \mathrm{bA}$ & $3.022,00 \mathrm{bA}$ & $1.953,00 \mathrm{bB}$ \\
\hline UEM 4 & $23,50 \mathrm{bA}$ & $20,99 \mathrm{bA}$ & $1.998,00 \mathrm{cA}$ & $858,00 \mathrm{~dB}$ \\
\hline UEM 5 & $29,87 \mathrm{aA}$ & $31,71 \mathrm{aA}$ & $3.502,00 \mathrm{bA}$ & $2.667,00 \mathrm{bB}$ \\
\hline UEM 6 & $30,33 \mathrm{aA}$ & $32,50 \mathrm{aA}$ & $2.926,00 \mathrm{bA}$ & $1.898,00 \mathrm{bB}$ \\
\hline UEM 7 & $28,16 \mathrm{aA}$ & $29,95 \mathrm{aA}$ & $3.030,00 \mathrm{bA}$ & $2.667,00 \mathrm{bA}$ \\
\hline
\end{tabular}

Médias seguidas de mesma letra minúscula na coluna não diferem entre si, pelo teste Scott-Knott $(\mathrm{P}>0,05)$. Médias seguidas de mesma letra maiúscula na linha não diferem entre si, pelo teste $\mathrm{F}(\mathrm{P}>0,05)$. 
Em relação a variável CE, os compostos UEM $2\left(30,87 \mathrm{~mL} \mathrm{~g}^{-1}\right)$, UEM $5\left(29,87 \mathrm{~mL} \mathrm{~g}^{-1}\right)$, UEM 6 $\left(30,33 \mathrm{~mL} \mathrm{~g}^{-1}\right)$ e UEM $7\left(28,16 \mathrm{~mL} \mathrm{~g}^{-1}\right)$ contiveram os maiores índices em Umuarama, não diferindo significativamente das testemunhas IAC $112(28,95$ $\left.\mathrm{mL} \mathrm{g}^{-1}\right)$, Zélia Original $\left(34,29 \mathrm{~mL} \mathrm{~g}^{-1}\right)$, BRS Angela $\left(26,41 \mathrm{~mL} \mathrm{~g}^{-1}\right)$, IAC $125\left(33,87 \mathrm{~mL} \mathrm{~g}^{-1}\right)$ e IAC 1283 $\left(32,29 \mathrm{~mL} \mathrm{~g}^{-1}\right)$. Esses novos compostos apresentam em sua constituição todos os genótipos comerciais supracitados e a incorporação de outros materiais de alta capacidade de expansão na sua formação é um forte indício da perspectiva de sucesso em relação aos ganhos de seleção para essa característica com os avanços das gerações no melhoramento intrapopulacional. Em Cidade Gaúcha os compostos UEM $5\left(31,71 \mathrm{~mL} \mathrm{~g}^{-1}\right)$, UEM $6\left(32,50 \mathrm{~mL} \mathrm{~g}^{-1}\right) \mathrm{e}$ UEM $7\left(29,95 \mathrm{~mL} \mathrm{~g}^{-1}\right)$, tiveram comportamento semelhante às testemunhas supracitadas além do genótipo Viçosa $\left(27,37 \mathrm{~mL} \mathrm{~g}^{-1}\right)$. Nesse sentido, os compostos superiores para a referida variável, podem ser úteis em programas de melhoramento, vez que tendem a alcançar ou até superar os mesmos níveis de qualidade que os materiais comerciais.

Segundo Nunes et al. (2002), a capacidade de expansão é característica extremamente importante na cultura do milho-pipoca, uma vez que, quanto maior for a $\mathrm{CE}$ da cultivar, maior será a qualidade da pipoca; uma vez que, melhora-se maciez e textura (SIMON et al., 2004). Nesse aspecto, para que o milho-pipoca possua valor comercial a CE deve ser igual ou superior a $30,00 \mathrm{~mL} \mathrm{~g}^{-1}$.

Os valores de $\mathrm{CE}$ dos compostos que se destacaram nesse estudo $\left(28,16\right.$ a $\left.32,50 \mathrm{~mL} \mathrm{~g}^{-1}\right)$ estão acima dos encontrados por Sawazaki et al. (1986), os quais observaram média de 17,50 $\mathrm{mL} \mathrm{g}^{-1}$. Em ensaio realizado em Campinas, SP, a CE variou de 18,40 a 28,80 $\mathrm{mL} \mathrm{g}^{-1}$ na cultivar RS-20 (FANTIN; SAWAZAKI; BARROS, 1991). Por sua vez, Merlo, Fornasieri Filho e Lam-Sánchez (1988) avaliando híbridos importados e nacionais verificaram valores de CE de 12,40 a 21,80 $\mathrm{mL} \mathrm{g}^{-1}$. Em contrapartida, os resultados aqui encontrados foram semelhantes aos obtidos por Galvão, Sawazaki e Miranda (2000) e Sawazaki et al. (2000), os quais verificaram bons resultados de CE em São Paulo e Minas Gerais.

$\mathrm{Na}$ avaliação de dialelo circulante para identificação de compostos a serem utilizados em programa de seleção recorrente intrapopulacional, Rangel et al. (2007) constataram que o melhor composto foi constituído pela reunião das populações UNB-2UC1 x BRS Angela com valores de capacidade de expansão (CE) e rendimento de grãos (RG) de $26,54 \mathrm{~mL} \mathrm{~g}^{-1}$ e $1.446,09 \mathrm{~kg} \mathrm{ha}^{-1}$, respectivamente.

Ainda em relação à $\mathrm{CE}$, verifica-se por meio da Tabela 3 que a maioria dos genótipos não revelou diferenças significativas $(\mathrm{P}>0,05)$ quando avaliados em locais diferentes, no caso, Umuarama e Cidade Gaúcha. Para Ruffato et al. (2000) a CE do milhopipoca pode ser de origem genética e não-genética, como as condições de desenvolvimento em campo, de colheita e do pré-processamento. Porém, nesta situação, os valores obtidos demonstram que a característica $\mathrm{CE}$ não sofreu interferência frente às condições ambientais. Provavelmente, este comportamento seja de origem genética e, desta forma, inerente a cada genótipo.

Em relação a variável RG (Tabela 3), verificase que os genótipos testados indicaram ampla variabilidade, com rendimentos de 4.012,00 $\mathrm{kg} \mathrm{ha}^{-1}$ a $883,00 \mathrm{~kg} \mathrm{ha}^{-1}$, em Umuarama. Observa-se ainda que IAC 125 (testemunha) e UNB 2 apresentaram RG médios maiores em relação aos demais, ao passo que nos genótipos avaliados em Cidade Gaúcha, os valores de RG variaram de 3.539,00 a $631,00 \mathrm{~kg} \mathrm{ha}^{-1}$.

Ao se comparar o rendimento dos genótipos entre os locais, observaram-se diferenças significativas. Por meio da Tabela 3, constata-se que as magnitudes de RG para os genótipos avaliados em Umuarama foram superiores àquelas expressas na Cidade Gaúcha. Cumpre destacar que não houve diferenças significativas para os genótipos IAC 112, Zélia Original, BRS Angela, IAC 1283, SC 002, PR-079, RR 046, SC 016, Compostos UEM 
1,UEM 2 e UEM 7, quanto ao RG entre os aludidos locais. Desse modo, o desempenho semelhante dos genótipos aqui destacados demonstra expressão de estabilidade biológica em ambas as condições de ambiente.

Por outro lado, o comportamento instável dos genótipos para $R G$ entre os dois locais pode ser justificado em função das oscilações do ambiente, principalmente déficit hídrico na fase de florescimento.

Em geral, o RG foi mais sensível em relação a $\mathrm{CE}$ entre os locais avaliados. Esses resultados contrariam as observações de Nunes et al. (2002), os quais, testando outras cultivares verificaram que a CE sofreu maior influência do ambiente do que o RG em condições ambientais desfavoráveis.

Os compostos UEM $3\left(3.022,00 \mathrm{~kg} \mathrm{ha}^{-1}\right)$, UEM 5 (3.502,00 kg ha-1), UEM $6\left(2.926,00 \mathrm{~kg} \mathrm{ha}^{-1}\right)$ e UEM $7\left(3.030,00 \mathrm{~kg} \mathrm{ha}^{-1}\right)$, produziram acima da média geral $\left(2.128,00 \mathrm{~kg} \mathrm{ha}^{-1}\right)$, mostrando, em relação ao grupo de genótipos avaliados, boa produtividade em Umuarama. Esses resultados, apesar de estarem abaixo dos apresentados pela testemunha IAC 125 e pela variedade UNB 2, não diferiram significativamente de IAC 1283 (testemunha). Por outro lado, o RG desses compostos foi superior a todos os demais genótipos, inclusive aos das demais testemunhas.

As médias de RG obtidas nesse trabalho estão acima da média de $2.766,00 \mathrm{~kg} \mathrm{ha}^{-1}$ da população com melhor desempenho (cv. Amarelo), em experimento instalado em Coimbra, MG, por Andrade et al. (2002). Conclui-se, pois, que as médias obtidas podem ser consideradas aceitáveis, vez que se trata de compostos.

Segundo Pacheco et al. (2000), em milho-pipoca a obtenção de variedade melhorada a partir de composto, tem se mostrado ser estratégia pertinente para o lançamento de variedades melhoradas. Como exemplo há a variedade BRS Angela, oriunda de ciclos de seleção recorrente no composto CMS43, da EMBRAPA-Milho e Sorgo. No entanto, as variedades melhoradas a partir de compostos são raras, mesmo sendo uma alternativa viável (SANTOS et al., 2008; VILELA et al., 2008).

Nos dois locais, o RG se mostrou de forma inconsistente em todos os genótipos que se destacaram para $\mathrm{CE}$, ou seja, nem sempre os materiais que se sobressaíram em relação ao $R G$ foram os que apresentaram maior índice de CE. No entanto, quando se avaliaram os compostos UEM 5, UEM 6 e UEM 7 verificou-se que estes apresentaram resultados satisfatórios tanto para $\mathrm{CE}$ quanto para RG. Por meio desses resultados, pode-se inferir que tais compostos são considerados promissores. Segundo Scapim et al. (2002), a síntese de compostos tem demonstrado ser uma estratégia eficiente em programas de melhoramento intrapopulacional com a finalidade de obter populações de milho-pipoca de boa produtividade e capacidade de expansão.

\section{Conclusões}

Os Compostos UEM 5, UEM 6 e UEM 7 apresentaram alta qualidade da pipoca (CE), com valores semelhantes às testemunhas em ambos os locais.

O rendimento dos genótipos foi mais instável do que a capacidade de expansão entre os locais avaliados.

Os compostos UEM 5, UEM 6 e UEM 7 apresentaram potencial satisfatório tanto para $\mathrm{CE}$ quanto para RG quando conduzidos em UmuaramaPR.

\section{Agradecimentos}

Os autores agradecem a Coodetec - Cooperativa Central de Pesquisa Agrícola, pela condução do experimento em Umuarama-PR. As sementes desses compostos estão disponíveis para todos os melhoristas do Brasil podendo entrar em contato com o Prof. Carlos Alberto Scapim. 


\section{Referências}

ANDRADE, R. A.; CRUZ C. D.; SCAPIM C. A.; SILVÉRIO L.; PINTO R. J. B.; TONET A. Análise dialélica da capacidade combinatória de variedades de milho pipoca. Acta Scientiarum. Agronomy, Maringá, v. 24, n. 5, p. 1197-1204, 2002.

CARPENTIERI-PÍPOLO, V.; TAKAHASHI, H. W.; ENDO, R. M.; PETEK, M. R.; SEIFERT, A. L. Correlações entre caracteres quantitativos em milho pipoca. Horticultura Brasileira, Brasília, v. 20, n. 4, p. 551-554, 2002.

CARPENTIERI-PÍPOLO, V.; SILVA, F.A. M.; SEIFERT, A. L. Popcorn parental selection based on genetic divergence. Crop Breeding and Applied Biotechnology, Viçosa, v. 3, n. 4, p. 261-268, 2003.

CRUZ, C. D.; CARNEIRO, P. C. S. Modelos biométricos aplicados ao melhoramento genético. Viçosa: Editora UFV, 2003.

CRUZ, J. C.; PEREIRA-FILHO, I. Milho: cultivares para 2008/2009. 2008. Disponível em: <http://www. cnpms.embrapa.br/milho/cultivares/index.php > Acesso em: 10 fev. 2009.

DAROS, M.; AMARAL JÚNIOR, A. T.; PEREIRA, M. G.; SANTOS, F. S.; GABRIEL, A. P. C.; SCAPIM, C. A.; FREITAS JUNIOR, S. P. Recurrent selection in inbred popcorn families. Scientia Agricola, Piracicaba, v. 61, n. 6, p. 609-614, 2004.

EMPRESA BRASILEIRA DE PESQUISA AGROPECUÁRIA - EMBRAPA. Centro Nacional de Pesquisa de Solos (Rio de Janeiro, RJ). Sistema brasileiro de classificação de solos. Brasília: EmbrapaSPI, Embrapa-CNPS, 1999. 412 p.

FANTIN, G. M.; SAWAZAKI, E.; BARROS, B. C. Avaliação de variedades de milho pipoca quanto à resistência a doenças e qualidade da pipoca. Summa Phytopathologica, Jaguariúna, v. 17, n. 2, p. 90-104, 1991.

FERREIRA, D. F. Sisvar, versão 4.3. Lavras: Universidade Federal de Lavras, 2003. 1 CD-ROM.

FREITAS JÚNIOR, S. P.; AMARAL JÚNIOR, A. T.; PEREIRA, M. G.; CRUZ, C. D.; SCAPIM, C. A. Capacidade combinatória em milho pipoca por meio de dialelo circulante. Pesquisa Agropecuária Brasileira, Brasília, v. 41, n. 11, p. 1599-1607, 2006.

GALVÃO, J. C. C.; SAWAZAKI, E.; MIRANDA, G. V. Comportamento de híbridos de milho pipoca em Coimbra, Minas Gerais. Ceres, Viçosa, v. 47, n. 270, p. 201-218, 2000.
GODOY, H.; CORREA, A. R.; SANTOS, D. Clima do Paraná. Manual agropecuário para o Paraná. Londrina: Fundação Instituto Agronômico do Paraná. 1976.

HALLAUER, A. R.; MIRANDA FILHO, J. B. Quantitative genetics in maize breeding. Ames: Iowa State University, 1988.

INSTITUTO AGRONÔMICO DO PARANÁ - IAPAR. Cartas climáticas básicas do Estado do Paraná. Londrina: Instituto Agronômico do Paraná, 1987.

MATTA, F. P.; VIANA, J. M. S. Testes de capacidade de expansão em programas de melhoramento de milho pipoca. Scientia Agricola, Piracicaba, v. 58, n. 4, p. 845851, 2001.

MERLO, E.; FORNASIERI FILHO, D.; LAMSÁNCHEZ, A. Avaliação de sete cultivares de milho pipoca (Zea mays L.) em três densidades de semeadura. Científica, São Paulo, v. 16, n. 2, p. 245-251, 1988.

MIRANDA, G. V.; SOUZA, L. V.; GALVÃO, J. C. C.; GUIMARÃES, L. J. M.; MELO, A. V.; SANTOS, I. C. Genetic variability and heterotic groups of Brazilian popcorn populations. Euphytica, Wageningen, v. 162, n. 3, p. 431-440, 2007.

NUNES, H. V.; MIRANDA, G. V.; GALVÃO, J. C. C.; SOUZA, L. V.; GUIMARÃES, L. J. M. Adaptabilidade e estabilidade de cultivares de milho pipoca por meio de dois métodos de classificação. Revista Brasileira de Milho e Sorgo, Sete Lagoas, v. 1, n. 3, p. 78-88, 2002.

PACHECO, C. A. P.; GAMA, E. E. G.; PARENTONI, S. N.; SANTOS, M. S.; LOPES, M. A.; FERREIRA, A. S.; FERNANDES, F. T.; GUIMARÃES, P. E. O.; CORREA, L. A.; MEIRELLES, W. F.; FELDMAN, R. O.; MAGNAVACA, R. BRS Angela: variedade de milho pipoca. Sete Lagoas: EMBRAPA/CNPMS. 2000. p. 1-6. (Comunicado Técnico, 27).

PEREIRA, M. G.; AMARAL JÚNIOR, A. T. Estimation of genetic components in popcorn based on nested design. Crop Breeding and Applied Biotechnology, Viçosa, v. 1, n. 1, p. 3-10, 2001.

PINTO, R. J. B.; SCAPIM, C. A.; BARRETO, R. R.; RODOVAlHO, M. A.; ESTEVES, N.; LOPES, A. D. Análise dialélica de linhagens de milho-pipoca. Ceres, Viçosa, v. 54, n. 315, p. 471-477, 2007.

RANGEL, R. M.; AMARAL JÚNIOR, A. T.; VIANA, A. P.; FREITAS JÚNIOR, S. P.; PEREIRA, M. G. Prediction of popcorn hybrid and composites means. Crop Breeding and Applied Biotechnology, Viçosa, v. 7, n. 3, p. 287-295, 2007. 
RANGEL, R. M.; AMARAL JÚNIOR, A. T.; SCAPIM, C. A.; FREITAS JÚNIOR, S. P.; PEREIRA, M.G. Genetic parameters and hybrids of circulant diallel in popcorn. Genetics and Molecular Research, Ribeirão Preto, v. 7, n. 4, p. 1020-1030, 2008.

RUFFATO, S.; CORREA, P. C.; MARTINS, J. H.; MANTOVANI, B. H. M.; SILVA, J. N. Efeito das condições de colheita, pré-processamento e armazenamento na qualidade do milho pipoca. Pesquisa Agropecuária Brasileira, Brasília, v. 35, n. 3, p. 591-597, 2000.

SANTOS, F. S.; AMARAL JÚNIOR, A. T.; FREITAS JÚNIOR, S. P.; RANGEL, R. M.; SCAPIM, C. A.; MORA, F. Genetic gain prediction of the third recurrent selection cycle in a popcorn population. Acta Scientiarum. Agronomy, Maringá, v. 30, n. 5, p. 651-658, 2008.

SAWAZAKI, E.; GALLO, P. B.; SORDI, G.; LONGO, L. S. Estudo da capacidade de expansão em cruzamentos dialélicos entre variedades de milho pipoca. In: CONGRESSO NACIONAL DE MILHO E SORGO, n. 15, 1986, Maceió. Anais... Maceió: EMBRAPA, DDT. 1986. p. $157-160$.

SAWAZAKI, E.; PATERNIANI, M. E. A. G. Z.; CASTRO, J. L.; GALlO, P. B.; GALVÃO, J. C. C.; SAES, L. A. Potencial de linhagens locais de milhopipoca para síntese de híbridos. Bragantia, Campinas, v. 59, n. 2, p. 143-151, 2000.

SCAPIM, C. A.; CARVALHO, C. G. P.; CRUZ, C. D. Uma proposta de classificação dos coeficientes de variação para a cultura do milho. Pesquisa Agropecuária Brasileira, Brasília, v. 30, n. 5, p. 683-686, 1995.

SCAPIM, C. A.; PACHECO, C. A. P.; TONET, A. T.; BRACCINI, A. L.; BARTH PINTO, R. J. Análise dialélica e heterose de populações de milho pipoca. Bragantia, Campinas, v. 61, n. 3, p. 219-230, 2002.

SCAPIM, C. A; BRACCINI, A. L; PINTO, R. J. B.; AMARAL JÚNIOR, A. T.; RODOVALHO, M. A.; SILVA, R. M.; MOTERLE, L. M. Componentes genéticos de médias e depressão por endogamia em populações de milho-pipoca. Ciência Rural, Santa Maria, v. 36, n. 1, p. 36-41, 2006.
SCOTT, A. J.; KNOTT, M. A. A cluster analysis method for grouping means in the analysis of variance. Biometrics, Raleigh, v. 30, n. 3, p. 507-512, 1974.

SIMON, A. G.; SCAPIM, C. A.; PACHECO, C. A. P.; PINTO, R. J. B.; BRACCINI, A. L.; TONET A. Depressão por endogamia em populações de milhopipoca. Bragantia, Campinas, v. 63, n. 1, p. 55-62, 2004.

VENDRUSCOLO, E. C. G.; SCAPIM, C. A.; PACHECO, C. A. P.; OLIVEIRA, V. R.; BRACCINI, A. L.; VIDIGAL, M.C. G. Adaptabilidade e estabilidade de cultivares de milho-pipoca na região centro-sul do Brasil. Pesquisa Agropecuária Brasileira, Brasília-DF, v. 36, n. 1, p. 123-130, 2001.

VENCOVSKY, R.; BARRIGA, P. Genética biométrica no fitomelhoramento. Ribeirão Preto: Sociedade Brasileira de Genética, 1992.

VIANA, J. M. S.; CONDÉ, A. B. T.; ALMEIDA, R. V.; SCAPIM, C. A.; VALENTINI, L. Relative importance of per se and topcross performance in the selection of popcorn $\mathrm{S}_{3}$ families. Crop Breeding and Applied Biotechnology, Viçosa, v. 7, n. 1, p. 74-81, 2007.

VIEIRA, R. A.; RODOVALHO, M. A.; SCAPIM, C. A., TESSMANN, D. J.; AMARAL JÚNIOR, A. T.; BIGNOTTO, L. S. Desempenho agronômico de novos híbridos de milho-pipoca no Noroeste do Estado do Paraná, Brasil. Acta Scientiarum Agronomy, Maringá, v. 30, n. 1, p. 29-36, 2009.

VILARINHO, A.; VIANA, J. M. S.; SANTOS, J. F.; CÂMARA, T. M. M. Eficiência da seleção de progênies $\mathrm{S}_{1}$ e $\mathrm{S}_{2}$ de milho pipoca visando à produção de linhagens. Bragantia, Campinas, v. 62, n. 1, p. 9-17, 2003.

VILELA, F. O.; AMARAL JÚNIOR, A. T.; PEREIRA, M. G.; SCAPIM, C. A.; VIANA, A. P.; FREITAS JÚNIOR, S. P. Effect of recurrent selection on the genetic variability of the UNB-2U popcorn population using RAPD markers. Acta Scientiarum. Agronomy, Maringá, v. 30, n. 1, p. 25-30, 2008. 
